\title{
A case of bilateral persistent sciatic arteries
}

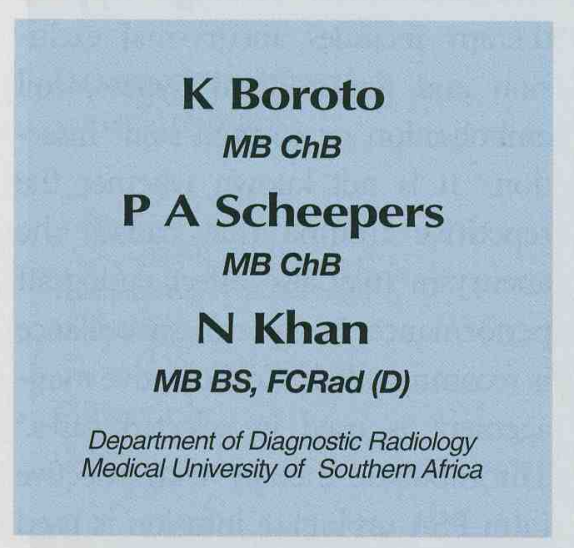

\section{Introduction}

Persistent sciatic artery (PSA) is a rare congenital arterial anomaly. Its various clinical signs may be explained by the special anatomy of the PSA. In this report we describe a patient presenting with bilateral pulsatile gluteal masses with associated symptoms of sciatic nerve compression worse on the left side.

\section{Case report}

A 44-year-old female patient presented with a 2-month history of bilateral gluteal masses, the left side bigger than the right, with associated sciatica more on the left. A fall on the right gluteal area prompted the consultation because of pain. There was no history of intermittent claudication. On examination, both gluteal regions appeared swollen, with the left bigger than the right. A distinct pulsatile mass was palpated on both sides.

Computed tomography (CT) scan of the pelvis was done and showed bilateral aneurysms which appeared to be originating from one of the gluteal vessels deep to the gluteal muscles, the left one being bigger and partially thrombosed (Figs 1 and 2). The patient subsequently had aneurysmal exclusion on the left side.

Conventional angiography of the lower limbs was only done 2 months after the above operation, and bilateral complete PSA with bilateral aneurysm was confirmed (Fig. 3). Postoperatively the left superficial femoral artery showed significant compensatory dilatation and had anastomosed with the popliteal artery just above the knee (Figs 4 and 5). The right superficial femoral artery ended in the distal thigh. Normal popliteal trifurcation was seen bilaterally.

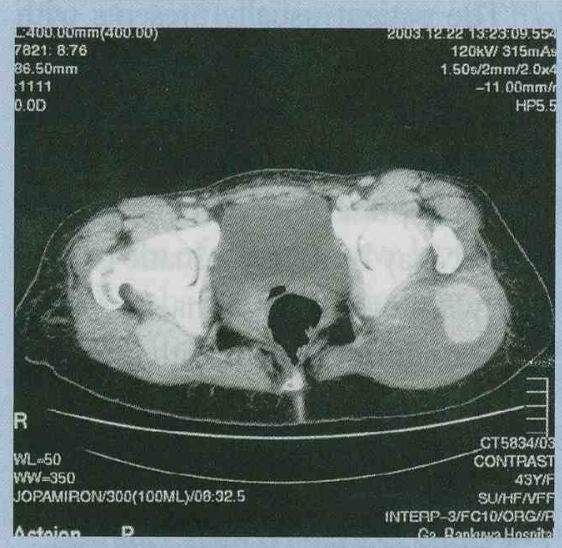

Fig. 1. Post-contrast axial CT scan through the pelvis showing bilateral aneurysmal dilatation of both PSAs, the left one being bigger and partially thrombosed.

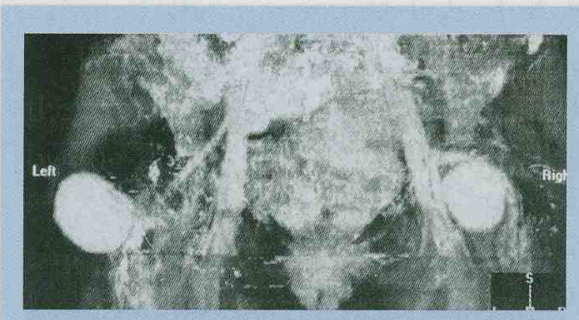

Fig. 2. $3 D$ reconstruction $C T$ angiogram of the pelvic vessels showing both aneurysms.

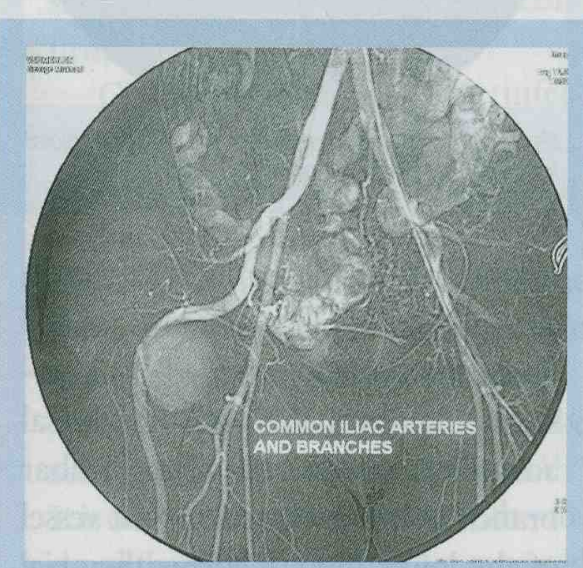

Fig. 3. Conventional angiogram of both common iliac vessels and branches. Aneurysmal dilation seen on the right PSA. Post-aneurysmal exclusion seen on the left.

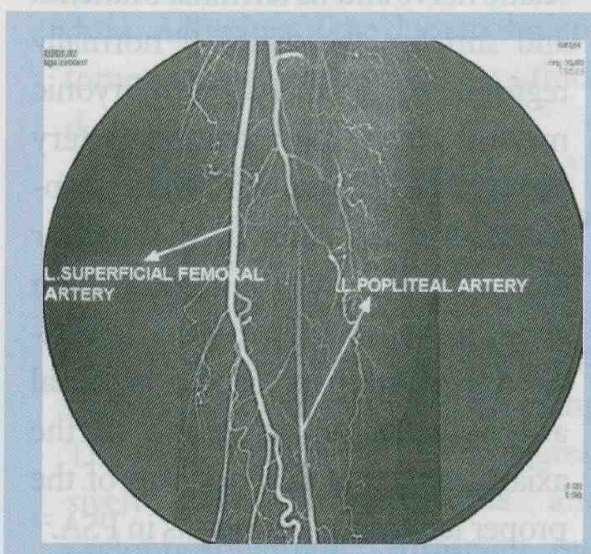

Fig. 4. Compensatory dilatation of the left superficial femoral artery that anastomoses distally with the popliteal artery.

\section{Discussion}

\section{Embryology and anatomy}

Extremities develop as buds from the trunk at 5 weeks of gestation, and by the end of the eighth week all- 


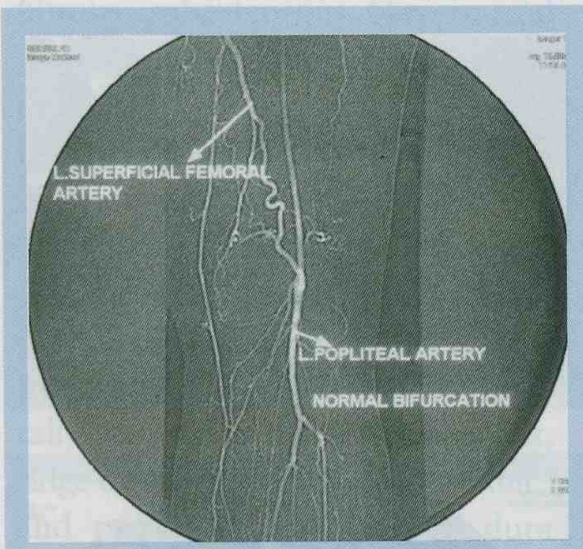

Fig. 5. Anastomosis between the left superficial femoral artery and left popliteal artery.

important arterial channels have been laid down. Vascular plexuses of the limbs are initially supplied by intersegmental branches of the dorsal aorta, of which the fifth lumbar branch becomes the dominant vessel of the lower limb. It forms the axial artery which passes in what eventually becomes the dorsal aspect of the limb. ${ }^{1}$ This artery accompanies the sciatic nerve and its terminal branches and supplies the digits. It normally regresses during the third embryonic month when the femoral artery forms. ${ }^{2}$ The original axial artery is represented in the adult by the inferior gluteal artery, which is a companion artery to the sciatic nerve and probably the distal part of the peroneal artery. Failure of regression of the axial artery or of development of the proper femoral artery results in PSA.,.3

PSA was first reported by Green in the Lancet in 1832. ${ }^{4}$ The incidence of PSA from a large series is $0.03 \%$ $0.06 \%$, the average age at presentation being 51 years. Aneurysmal dilatation is seen in $25 \%$ of cases, usually at the level of the trochanters. ${ }^{1}$ Twenty per cent to $33 \%$ of aneurysms are bilateral. ${ }^{1,2}$

The high incidence of aneurysmal formation is most probably due to repeated external trauma since PSA occupies a vulnerable position. Other causes postulated include accelerated atherosclerosis and a congenital lack of arterial wall elastic tissue. ${ }^{3}$ Nthle et al. ${ }^{4}$ proposed that the limited osseofibromuscular space of the greater sciatic foramen below the pyriformis also constricts this vessel, resulting in post-stenotic dilation and aneurysm formation.

PSA is prone to early arteriosclerotic changes and aneurysmatic degeneration that can complicate with thrombotic occlusion or peripheral embolism. PSA is associated with other congenital anomalies including Mullerian and renal agenesis, arteriovenous fistula, bone or soft tissue hypertrophy/hypotrophy, multiple angioma and neuroma. ${ }^{1,2}$ Coexistent venous abnormalities are extremely rare. ${ }^{2}$ The first case of PSA as an important collateral to an occluded ilio-femoral system was published in July $2000{ }^{3}$

The patient usually presents with pulsatile gluteal masses associated with sciatica and rarely with absent femoral pulse and normal palpable popliteal pulse (Cowie's sign). ${ }^{2}$ The patient may be asymptomatic or present with lower limb ischaemia, typically on sitting down or with intermittent claudication. ${ }^{5}$

Arteriography is the prime diagnostic method. ${ }^{5}$ Doppler ultrasound, CT angiography, and magnetic resonance angiography are useful in patients with arterial occlusion and have the advantage of combining cross-section and angiographic examination. CT angiography also details the relationship of the artery to bony landmarks. ${ }^{5,6}$

Treatment depends on symptoms and anatomical findings. Applied therapy includes aneurysmal exclusion and femorodistal bypass, coil embolisation or covered stent insertion. ${ }^{6}$ It is not known whether the repetitive trauma that causes the aneurysm may also affect endograft performance. Long-term surveillance is recommended. ${ }^{7}$ Conservative management is used in selected cases. ${ }^{6}$ Thrombolytic therapy with selective intra-PSA urokinase infusion is used in acute leg ischaemia Treatment of asymptomatic PSA without aneurysm formation is controversial. It should probably be left alone and the patient followed up?

\section{References}

1. Hassan A, Symptomatic persistent sciatic artery. J Am Coll Surg 2004; 199: 171-173.

2. Parry DJ, Munther I, Aldoori RJ, et al. Persistent sciatic vessels, varicose veins and lower limb hypertrophy: An unusual case or discrete clinical syndrome? J Vasc Surg 2002; 36: 396-400.

3. Bachelor TJP, Vowden P. A persistent sciatic artery aneurysm with an associated internal iliac artery aneurysm. Eur I Vasc Endovasc Surg 2000; 20: 400-402.

4. Nthe LM, Ghoor F, Blumberg L. Sciatic artery aneurysm. S Afr J Surg 1994; 32: 102-105.

5. Brancaccio G, Pera EFM, Celoria G, Stefanini T, Puccianti F. Symptomatic persistent sciatic artery. J Am Coll Surg 2004; 198: 158.

6. Aschwanden EJM, Heidecker HG. Thalhammer C, Jaeger KA, Bilec D. Acute onset of intermittent claudication caused by complete occlusion of a persistent sciatic artery. Eur J Vasc Endovasc Surg Extra 2004; 7: 61-63.

7. Samson RH, Showalter DP Persistent sciatic artery as collateral for an occluded iliofemoral system. J Vasc Surg 2004; 40: 183. 